\title{
A New Type of Fibergastroscope (FGS-BLG) for Measurement of Gastric Lesions
}

\author{
Masataka Maruyama, Shisho Ichioka, Tadayoshi Takemoto \\ and TaIgoro Kondo \\ Department of Gastroenterological Medicine, Tokyo Women's \\ Medical College, Tokyo \\ Shoichi Yamagata and Kotaro Ueno \\ Department of Medicine, Tohoku University School of Medicine, \\ Sendai \\ Kenji Tsuneoka and Masahiko Sesoko \\ Department of Medicine, Nippon Medical College, Tokyo
}

\begin{abstract}
Maruyama, M., Ichoka, S., Takemoto, T., Kondo, T., Yamagata, S., Ueno, K., Tsuneora, K. and Sesoko, M. A New Type of Fibergastroscope (FGS-BLG) for Measurement of Gastric Lesions. Tohoku J. exp. Med., 1972, 107 (2), 159165 - An attempt to measure a lesion in the stomach through a new model of fibergastroscope (FGS-BLG) is reported. FGS-BLG is equipped with the gauge which is observed as a bright line above the image on view of the scope. The length of this line always corresponds with the length of $1 \mathrm{~cm}$ on the surface of the gastric mucosa on which the object lens is just focused. The effective range of the distance between the lesion and the object lens is approximately 2 to $E \mathrm{~cm}$, although the lesions near the pyloric ring and on the posterior wall of the upper portion of the corpus are not always satisfactorily measured because the distance is generally too long and too short respectively. The gauge is easy tc photograph, so that the size of lesion in the stomach can be satisfactorily measured even on the developed picture. This device will be widely applicable. fibergastroscope; gastric diseases
\end{abstract}

The endoscopic diagnosis of gastric diseases has advanced so remarkably that it is possible nowadays to detect early cancer of the stomach, even less than $1 \mathrm{~cm}$ in size. However, the more minute endoscopic observation is desirable. Suitable extension of the gastric mucosa under regulated air pressure in the gastric lumen and the measurement of a lesion in the stomach under direct vision should be a help for such minute endoscopic observation. In the former attempt, a model designed to regulate intragastric pressure constantly was reported by Yamada et al.*

This paper deals with a new type of fibergastroscope to measure a lesion in the stomach together with clinical experiences.

Received for publication, Appril 19, 1971.

* Yamada K. et al. reported on the 11th Kanto Regional Meeting of Japanese Society for Endoscopy, 1971. 


\section{Methods and Materials}

A new type of fibergastroscope, which was made by Machida Co. Ltd., was designed to measure a lesion in the stomach. The structure of this new model (FGS-BLG) is almost identical with FGS-BL type fibergastroscope for biopsy, except for a difference at the tip of the fiberscope as shown in Fig. 1. FGS-BLG has an oblong window for gauge beside the window for observation. The light entering from here is observed as a bright line above the image on view as shown in Figs. 5 and 6 . The length of this line indicates the length of $1 \mathrm{~cm}$ on the surface of the gastric mucosa on which the object lens is just focussed. FGS-BLG is equipped with an wing-like plate beside the series of object lens incorporated at the tip (Fig. 2). This plate shifts along with the movement of the series of object lens in the process of focussing, interrupting the light coming through the oblong window into the fiber bundle for gauge according to the distance between the object lens and the lesion. The length of gauge above the image on view corresponds to the length of $1 \mathrm{~cm}$ on the surface of a lesion.

Accuracy of the measurement of lesion through FGS-BLG was evaluated by measuring a model scale, and the lesions of gastric ulcer and gastric polyp were clinically measured.

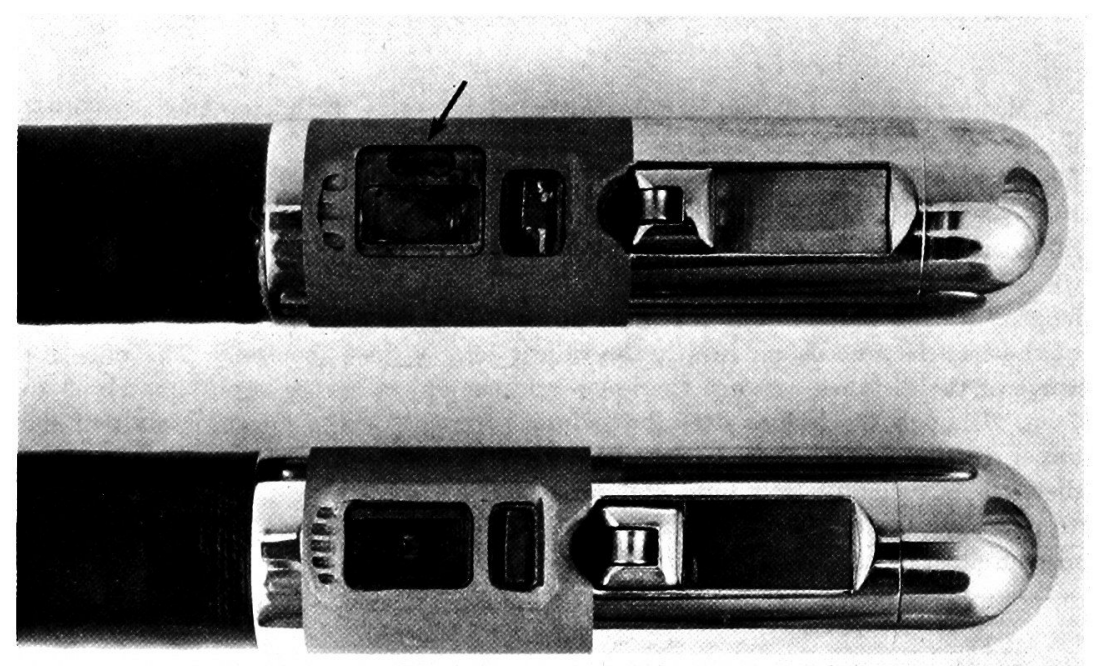

Fig. 1. The tip of FGS-BLG (upper) and FGS-BL (lower). FGS-BLG has an oblong window for gauge (arrowed) beside the window for image.

\section{Results}

Photographs of a model scale graduated in mm were taken through FGS-BLG as shown in Fig. 3. In each picture from $3 \mathrm{a}$ to $3 \mathrm{~h}$, the distance between the scale and the object lens was changed from $1 \mathrm{~cm}$ to $8 \mathrm{~cm}$ at an interval of $1 \mathrm{~cm}$. When the distance between the scale and the object lens was less than $1 \mathrm{~cm}$, the light from the oblong window came into the fiber bundle for gauge without interruption by the light-blocking plate, and the length of gauge did not change any longer. When the distance was more than $1 \mathrm{~cm}$, the length of gauge almost corresponded with $1 \mathrm{~cm}$ of the scale as shown in Fig. 3 . At a distance of more than $8 \mathrm{~cm}$, 


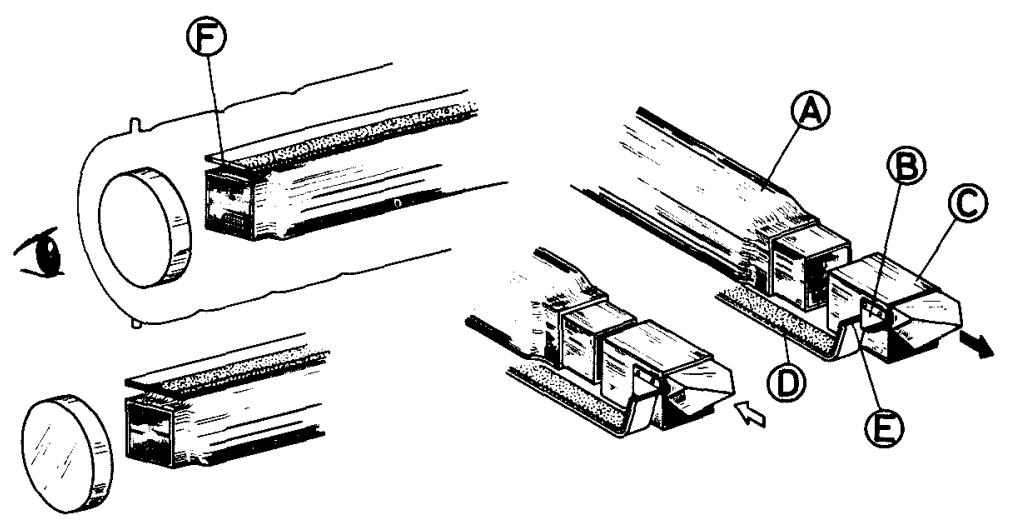

Fig. 2. The structure of FGS-BLG.
A: fiber bundle for image
$C$ : object lens
$\mathrm{E}$ : entrance of light for gauge
$B$ : wing-like plate for blocking light
D: fiber bundle for gauge
$\mathrm{F}$ : gauge (scale)
Light coming into the fiber bundle for gauge (D) is cut off by the wing-like plate (B) in proportion to object-lens distance.

however, a possibility of error for comparative measurement of the object seemed to increase as the length of gauge itself became too short. In addition, the focus depth becomes deeper as the distance becomes greater. As shown in Fig. 4, relationship between the distance (lens-object) and the length of gauge measured in each photograph in Fig. 3 showed an exponential curve, which elucidated an inverse relation between the length of gauge and the object-lens distance. Therefore, it is considered that the length of gauge itself could be a source of error when the lens-object distance becomes longer. The effective range of the distance between a lesion and the object lens is thus limited to approximately 2 to $5 \mathrm{~cm}$ for reliable measurement.

Consequently, the effective range for intragastric measurement by FGS-BLG is inevitably limited. It was inadequate to measure a lesion near the pyloric ring to which the tip of FGS-BLG cannot always approach and that on the posterior wall of the corpus and the cardia in which the distance tends to be too short. The size of lesion to be measured is also limited when the distance is short. It was not possible to measure precisely the lesion which was too large to be seen in one field of view unless the distance of more than $5 \mathrm{~cm}$ was maintained. Hence, the lesion below $4 \mathrm{~cm}$ in size appeared to be most suitable for this method.

Approximately 450 cases of ulcer and polyp on various portions of the stomach were subjected to this method, and the results were satisfactory in most cases. The gauge above the picture of an ulcer in Fig. 5 agreed fairly well with the scale graduated in $5 \mathrm{~mm}$ which was placed in the stomach. In this measurement, the major diameter of the ulcer in Fig. 5 was approximately $1.2 \mathrm{~cm}$, and the minor diameter was approximately $0.8 \mathrm{~cm}$. It was rather difficult to measure such protruded lesions as polyp at a short distance, since the focus depth 


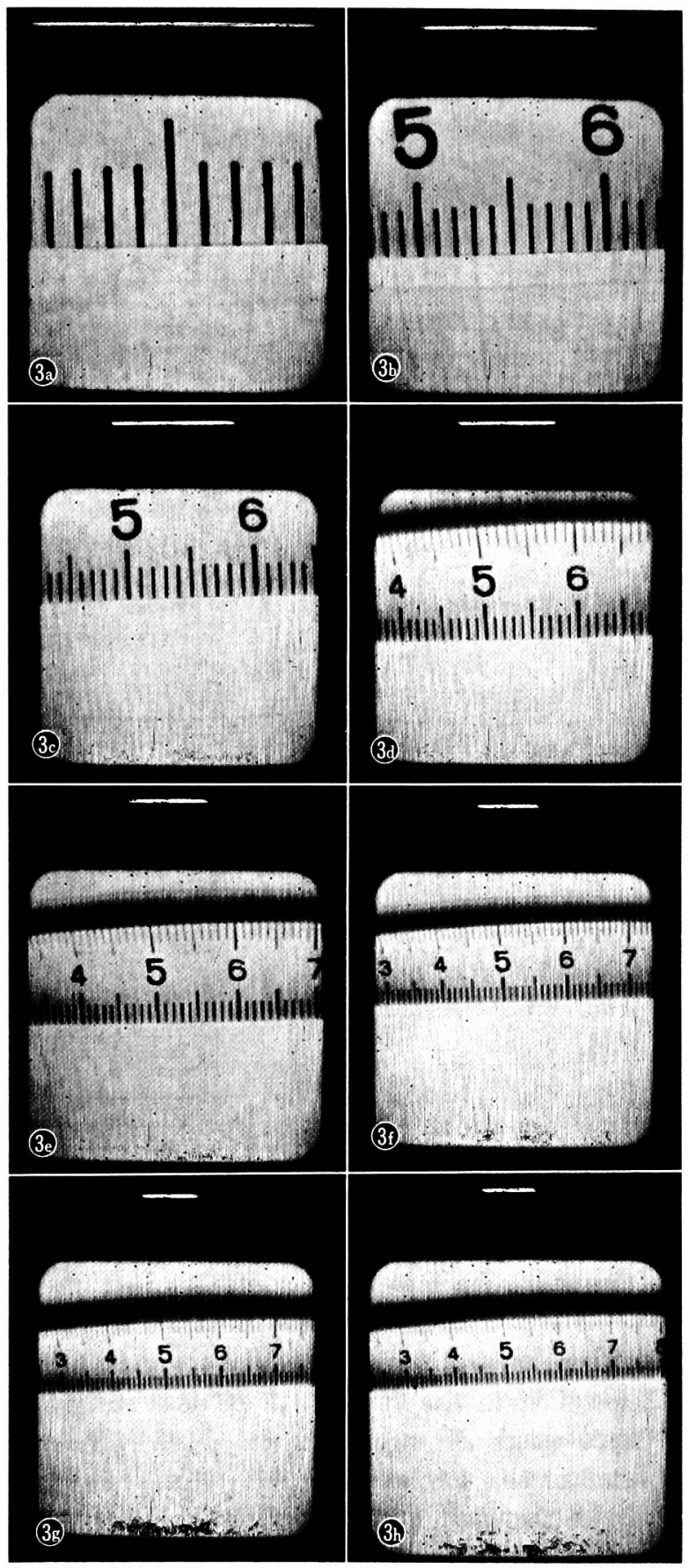

Fig. 3. Photographs of a model scale graduated in $\mathrm{mm}$ taken through FGS-BLG. From (a) to (h), the distance between the scale and the object lens changed from $1 \mathrm{~cm}$ to $8 \mathrm{~cm}$ at an interval of $1 \mathrm{~cm}$. 


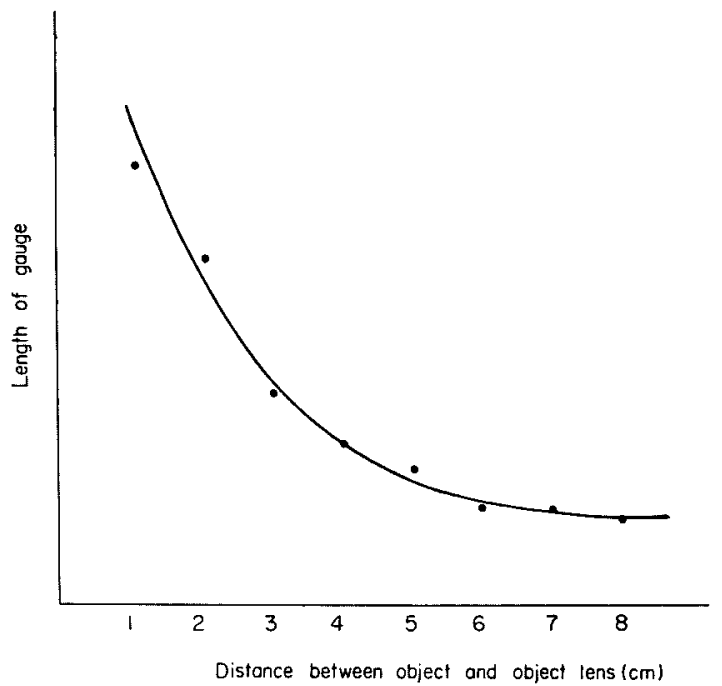

Fig. 4. Relationship between the object-lens distance and the length of the gauge.

became short and the length of gauge changed too much even upon minor transposition of the point on which the object lens was focused. When the object lens was focussed on the most proximal surface of the polyp as shown in Fig. 6a, the length of gauge indicated the length of $1 \mathrm{~cm}$ there-on but this was longer than $1 \mathrm{~cm}$ in the actual size of the polyp. In cases of polyp, therefore, the object lens should be focussed on the edge of polyp even though the picture is blurred as shown in Fig. 6b. The polyp thus measured in Fig. 6b was approximately $1.3 \mathrm{~cm}$ in diameter, which almost corresponded with the actual size as shown in Fig. 7.

\section{Discussion}

Attempts were made to measure the lesions in the stomach. Kimura and Takemoto (1970) tried to put a scale, just like the one we used in Fig. 5, into the stomach through the route of biopsy forceps in FGS-BL to settle the place of biopsy at a certain distance from the border of the area of fundic gland. Karasawa et al. $(1970)^{*}$ presented a tentative model of stereoscopic gastrocamera for measurement of lesion in the stomach. But, in practice, it is quite difficult to place the scale at the appropriate position in the stomach. At any rate, stereoscopic gastrocamera has some unsolved problems, although it is a good idea. On the other hand, in our method, the gauge is easy to photograph, so that the lesion in the stomach can be satisfactorily measured even on the developed picture. So this device will be widely applicable.

\section{Reference}

1) Kimura, K. \& Takemoto, T. (1970) Biopsy in chronic gastritis. Stomach and Intestine, (Jap.), 5, 843-851.

\footnotetext{
* Karasawa, Y. et al. reported at the 10th Kanto Regional Meeting of Japanese Society for
} Endoscopy, 1970. 
Fig. 5. Photographs of an ulcer at the angulus of the stomach.

Fig. 6. Photographs of a polyp in the antrum.

(a) is just focused on the proximate surface of the polyp, and

(b) on the edge of the polyp.

Fig. 7. Photograph of the same polyp as in Fig. 6 taken after gastrotomy. 

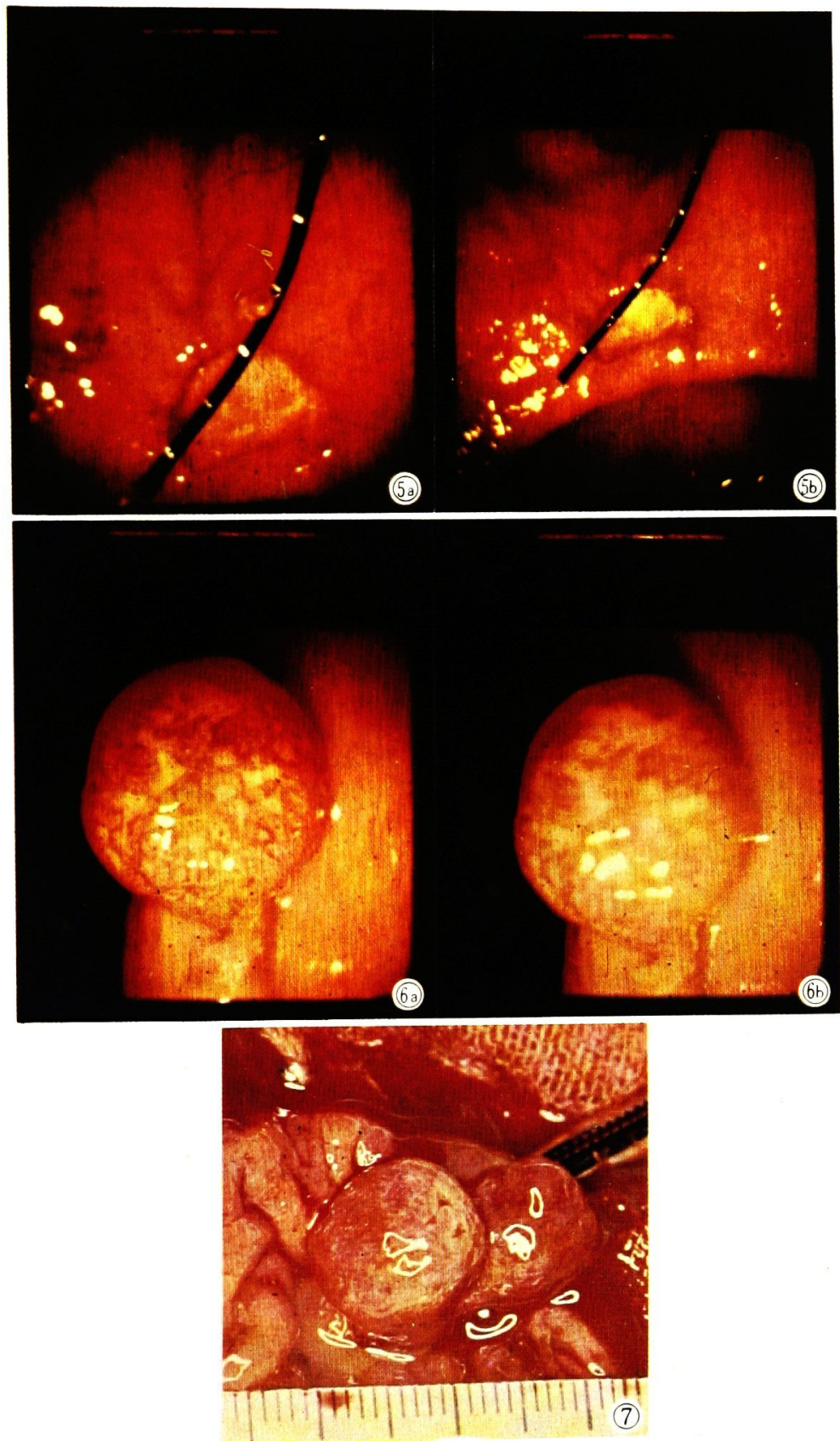